This item was submitted to Loughborough's Research Repository by the author.

Items in Figshare are protected by copyright, with all rights reserved, unless otherwise indicated.

\title{
Optimal management of reactive power sources in far-offshore wind power plants
}

PLEASE CITE THE PUBLISHED VERSION

https://doi.org/10.1109/PTC.2017.7980833

PUBLISHER

(C) IEEE

VERSION

AM (Accepted Manuscript)

LICENCE

CC BY-NC-ND 4.0

\section{REPOSITORY RECORD}

Theologi, A.M., M. Ndreko, Jose L. Rueda, M.A.M.M. Van Der Meijden, and Francisco M. Gonzalez-Longatt. 2019. "Optimal Management of Reactive Power Sources in Far-offshore Wind Power Plants". figshare. https://hdl.handle.net/2134/25770. 


\title{
Optimal Management of Reactive Power Sources in Far-offshore Wind Power Plants
}

\author{
A.M. Theologi ${ }^{1,2}$, M. Ndreko $^{1}$, J.L. Rueda ${ }^{1}$, M.A.M.M. van der Meijden ${ }^{1,3}$, F. González-Longatt ${ }^{4}$ \\ ${ }^{1}$ Delft University of Technology, The Netherlands \\ ${ }^{2}$ Aristotle University of Thessaloniki, Greece \\ ${ }^{3}$ TenneT TSO B. V, The Netherlands \\ ${ }^{4}$ Loughborough University, United Kingdom
}

\begin{abstract}
This paper introduces a new approach for the optimal management of reactive power sources, which follows a predictive optimization scheme (i.e. day-ahead, intraday application). Predictive optimization is based to the principle of minimizing the real power losses, as well the number of On-load Tap Changer (OLTC) operations for 24 time steps ahead. The mixed-integer nature of the problem and the restricted computing budget is tackled by using an emerging metaheuristic algorithm called Mean-Variance Mapping Optimization (MVMO). The evolutionary mechanism of MVMO is enhanced by introducing a new mapping function, which improves its global search capability. The effectiveness of MVMO (i.e. fast convergence and robustness against randomness in initialization and factors used in evolutionary operations) and the achievement of optimal grid code compliance are demonstrated by investigating the case of a far-offshore wind power plant, interconnected with HVDC link.
\end{abstract}

Index Terms-optimal reactive power management, meanvariance mapping optimization, on-load tap changer

\section{INTRODUCTION}

Offshore wind is a competitive power source and increasingly attractive investment with various benefits for the electric power generation. Europe is considered as the frontrunner in this field, where during the year 2015 new offshore capacity of $3.02 \mathrm{GW}$ was connected to the grid [1]. According to wind energy scenarios for 2030, offshore wind installations amount to $66 \mathrm{GW}$ [2]. However, the high penetration of the wind power into the energy systems holds many technical/operational challenges. Offshore wind power plants are required to provide reactive power support during both the steady-state as well as during AC fault conditions [3].

Nowadays, the Transmission System Operators (TSO) of each country have defined Grid Code Requirements in order to ensure the safe, secure and reliable operation of power systems. Traditionally, the reactive power sources in the synchronous transmission systems are designed for operation in an uncoordinated manner, i.e. meeting local targets as seen at the terminal bus of each device. Although the reactive power requirement at the point of common coupling (PCC)

This work was supported by Delft University of Technology. can be achieved without major drawbacks, the aforementioned traditional approach is quite conservative (i.e. it does not entail efficient and optimal management of the reactive power sources) [4]. Since it is highly related to the way how the active and reactive power flow through a give grid topology, the optimal reactive power management is a particular form of optimal power flow (OPF) and a subject of remarkable research, which has immense significance on the security and economical operation of the power systems (e.g. by minimizing losses and ensuring fulfilment of technical constraints) [5], such as the control and optimization for operation of wind power plants [6].

This paper proposes an approach that involves coordinated management of reactive sources based on an OPF formulation that accounts for non-linear power flow equations. The benefit brought is minimum power losses and reduction of stress or disturbances for the controllable devices, i.e. transformers, simultaneously [4]-[7]. In order to solve this problem mathematically, various optimization algorithms that have been developed so far could be applied in principle, since the existing technologies for data communication and acquisition render the coordinated management as a feasible task. However the classical optimization algorithms, such as gradient-based algorithms, described in [8]-[10], struggle with non-linearity and nonconvexity of the problem, which is also characterized by discontinue and multimodal landscape [11]. Conclusively, the classical optimization tools are not flexible to be applied in a complex search space and are sensitive to the initial points as well [12].

Classical heuristic optimization algorithms, like genetic algorithms, particle swarm optimization, differential evolution, and evolutionary strategies, constitute alternative tools to tackle the above indicated optimization problems. Nevertheless, the effectiveness of these algorithms is highly dependent on finding proper parameter settings and usually entails significant algorithmic modifications. In addition, due to their population based search framework, these algorithms are not suitable for online applications, in which it is crucial to find optimal solutions within very reduced computing 
budget. For avoiding unwanted occurrences, such as local stagnation or premature convergence, this reliance should be taken into consideration [12], [11]. As a result, nowadays, the research interest focus on new metaheuristic solutions, such as mean-variance mapping optimization (MVMO) [13], linearized biogeography-based optimization (LBBO) [14], firework algorithm (FWA), firefly algorithm (FA) cuckoo search (CS) [15], bat algorithm (BA) [16] and teachinglearning-based optimization (TLBO) [17], which, due to their conceptual simplicity, can be easy adapted without significant modifications.

Unlike the majority of existing and popular metaheuristic algorithms, MVMO can be configured to evolve a single solution (single parent-offspring approach) throughout the search process. This is an advantage in terms of computing effort (i.e. less amount of problem evaluations), but might increase the risk of premature convergence. Nevertheless, this challenge is addressed in this paper by exploring the use of a new mapping function, which aims at improving the ability of MVMO to strategically switch between search exploration (i.e. generating diverse solutions in an attempt to cover the whole search space) and search exploitation (i.e. intensifying the search in a specific region of the search space), thus improving the global search capability of the algorithm.

The remainder of this paper is organized as follows. Section II gives an overview of the proposed optimization approach and describes the MVMO-based procedure. In Section III, a test case is developed and evaluated. Finally, conclusions and outlook for future work are presented in Section IV.

\section{PROPOSED APPROACH}

Fig. 1 depicts schematically the structure of the proposed approach. The optimization is performed for a given scenario, which includes a set of future operating points on a 24-hour time horizon [7]. The predicted wind speed for the considered time period results directly form a Neural Network (NN) based wind speed forecasting [18] and is received by the optimization algorithm as input. Then, MVMO suggests the optimal OLTC tap settings of the offshore transformers together with the optimal reactive power reference for each wind turbine.

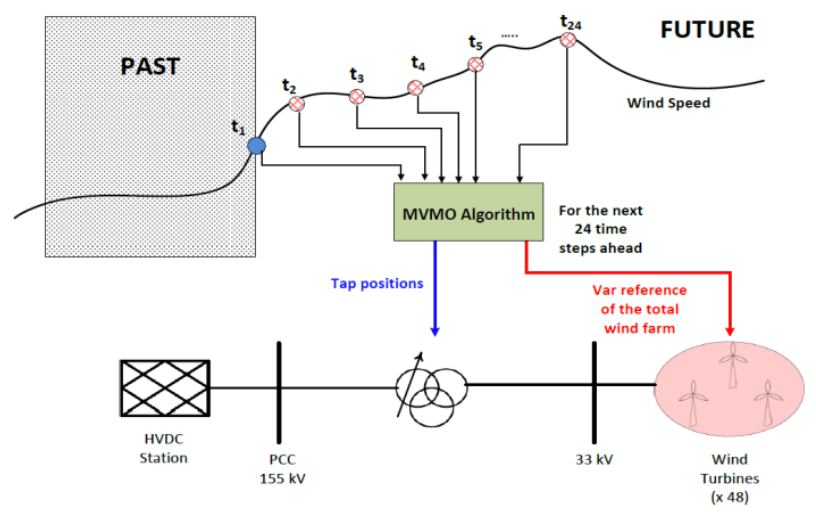

Figure 1. Predictive optimization approach.

\section{A. Optimization problem statement}

Considering the total real power losses and the operation cost of the OLTC on a 24-hour time horizon, the formulation of the objective function is multi-objective and is given by the following equation, in which the problem is treated as single objective due to the use of the weight coefficients.

Minimize

$$
O F=\sum_{t=1}^{24}\left(w_{1} \cdot P_{L, t}+w_{2} \cdot O L T C_{c o s t, t}\right)
$$

subject to,

$$
\begin{aligned}
& v_{\text {min }} \leq v \leq v_{\text {max }} \\
& i \leq i_{\text {lim }} \\
& s \leq s_{\text {lim }}
\end{aligned}
$$

where, $t$ stands for the time index and $P_{L, t}$ constitutes the hourly real power losses. The system operating constraints given by (2)-(4) constitute the inequality constraints on the dependent variables, such as the voltage magnitude of the buses, the current through the cables, line and transformer flow limits, respectively.

The bounds of decision variables refer to the wind turbines Var settings and the transformers tap change limits. They define the search space for the optimization algorithm and are described by the following equations:

$$
\begin{gathered}
q_{W T G}^{\min } \leq q_{W T G} \leq q_{W T G}^{\max } \\
\operatorname{tap}_{T r, \text { min }} \leq \operatorname{tap}_{T r} \leq t a p_{T r, \text { max }}
\end{gathered}
$$

The hourly operation cost denoted by $O L T C_{\text {cost }, t}$ is stated as follows:

$$
\text { OLTC }_{\text {cost }, t}=w_{3} \cdot\left|\operatorname{tap}_{t}-\operatorname{tap}_{t-1}\right|
$$

where $\operatorname{tap}_{i}$ stands for the discrete tap positions at hour $\mathrm{t}$ and $\mathrm{t}$ 1 , and $w_{1-3}$ are the weight coefficients corresponding to cost values, which were set to $w_{1}=80, w_{2}=10$, and $w_{3}=1$.

\section{B. $M V M O$}

MVMO belong to the family of evolutionary optimization algorithms and can be applied in multi-objective mixed integer and non-linear problems. Remarkably, it can be configured to perform to evolve a single solution throughout the optimization process or as a population-based; the first option is chosen in this paper, since it is intended for online application. The proposed methodology, based on MVMO as solver, for the formulated optimal reactive power management is described in Fig. 2 [4]. The procedure starts with the initialization of the parameter settings, such as the archive size, the selection method for evolution of optimization variables, and the maximum number of iterations. The searching space of all variables is confined in $[0,1]$ and therefore the real $\mathrm{min} / \mathrm{max}$ have to be normalized to this interval. Therefore, during every iteration step, it is guaranteed that the solution vector does not violate the required boundaries [7].

Remarkably, MVMO bases its evolutionary mechanism on a special mapping function that extracts the statics successful behavioral pattern of the evolved solutions as described by 
mean and shape variables. This information is used to transform a variable $x_{i}^{*}$ varied randomly with unity distribution to another variable $x_{i}$. Besides, after each fitness evaluation, a solution archive is filled and continuously updated throughout the search. The archive stores and ranks the most successful solutions achieved so far [19]. The evolutionary loop is performed until a specified termination criterion is met (e.g. maximum number of function evaluations) [12]. The different stages of MVMO-based procedure are presented in the following paragraphs.

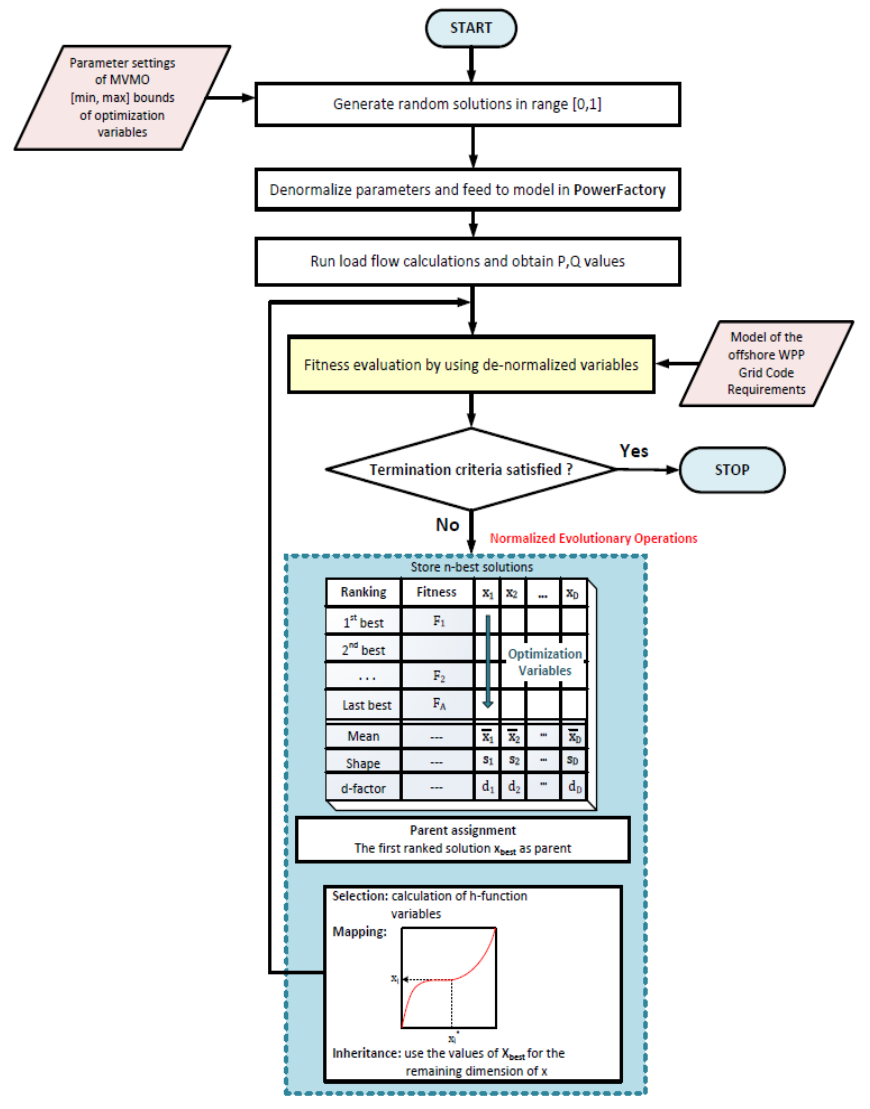

Figure 2. MVMO-based procedure for optimal reactive power management

1) Initialization - The initial candidate solution is randomly generated between the boundaries as follows:

$$
x_{i}^{\text {init }}=x_{i}^{\text {min }}+\operatorname{rand}\left(x_{i}^{\text {max }}-x_{i}^{\text {min }}\right), \quad i=1,2, \ldots, D
$$

The index $i=1,2, \ldots, \mathrm{D}$ concerns with the problem dimension, so $\mathrm{D}$ is the number of decision variables. In this case, in which the optimizatin is performed in a predictive manner, after the first hour of the day, the intial candidate solution for the subsequent hours is generated by the best solutions obtained from the previous hour.

2) Fitness Evaluation \& Local Search - Before the fitness evaluation is performed, the decision variables are denormalized from the interval $[0,1]$ to the original [min, max] boundaries. The normalized range, within which MVMO performs, ensures there is no violation of bound constraints. Finally, after the termination criterion is satisfied, which is specified in this paper as a predefined number of fitness evaluations, the search process stops. Alternatively, in case that there is no improvement of fitness over successive fitness evaluations, then the process can be also terminated. In order to instensify the search once MVMO has found an attractive region, local search strategy, e.g. subordinating other classical or heuristc algorithms, can be added into the fitness evaluation stage.

3) Solution archive- The solution archive, where the $n$ best individuals obtained so far by MVMO are stored, serves as the knowledge base for guiding the algortihm's searching direction. The size of the solution archive remains constant for the entire process and is set in the intialization stage. The filling of the archive obey to a descending order of fitness over the iterations as presented in Fig. 3 and consequently, the oveall best found so far is always the first ranked solution. Once the archive is full, an update is donducted only if the solution fitness evaluation revealed that the new solution is better than those already stored in the archive. Since the fitness improves over the iterations, the stored solutions in the archive keep changing.

\begin{tabular}{|c|c|c|c|c|c|}
\hline Ranking & Fitness & $\mathrm{x}_{1}$ & $\mathrm{x}_{2}$ & $\ldots$ & $\mathrm{x}_{\mathrm{D}}$ \\
\hline $1^{\text {* }}$ best & $\mathrm{F}_{1}$ & & & & \\
\hline $2^{\text {ed }}$ best & & & \multicolumn{2}{|c|}{$\begin{array}{c}\text { Normallized } \\
\text { Optimization } \\
\text { Variables }\end{array}$} \\
\hline$\cdots$ & $\mathrm{F}_{2}$ & & \multicolumn{2}{|c|}{} \\
\hline Last best & $\mathrm{F}_{\mathrm{A}}$ & & & & \\
\hline \hline Mean & $\cdots$ & $\overline{\mathrm{x}}_{1}$ & $\overline{\mathrm{x}}_{2}$ & $\cdots$ & $\overline{\mathrm{x}}_{\mathrm{D}}$ \\
\hline Shape & $\cdots$ & $\mathrm{s}_{1}$ & $\mathrm{~s}_{2}$ & $\cdots$ & $\mathrm{s}_{\mathrm{D}}$ \\
\hline d-factor & $\cdots$ & $\mathrm{d}_{1}$ & $\mathrm{~d}_{2}$ & $\cdots$ & $\mathrm{d}_{\mathrm{D}}$ \\
\hline
\end{tabular}

Figure 3. Solution archive

The mean and shape variables are computed after every update of the archive for each optimization variable as follows:

$$
\begin{gathered}
\bar{x}_{i}=\frac{1}{n} \sum_{j=1}^{n} x_{i}(j) \\
s_{i}=-\ln \left(v_{i}\right) \cdot f_{s}
\end{gathered}
$$

where, the variance is calculated only for different variables in the archive by usin (10).

$$
v_{i}=\frac{1}{n} \sum_{j=1}^{n}\left(x_{i}(j)-\overline{x_{\imath}}\right)^{2}
$$

At the beginning $v_{i}$ is set to 1 , since $\overline{x_{l}}$ corresponds with the initialized value of $x_{i}$. The computed shape variable $s_{i}$ is one of the mapping function inputs with strong influence on its geometric characteristic shape. For this reason, the scaling factor $f_{s}$, which allows controlling the form of the mapping function and the search process, is involved in the calculation of $s_{i}$. 
4) Offspring generation- To create a new solution, MVMO uses a random sampling strategy. In order to generate a new solution, in every iteration the solution with the best fitness so far is used. It is assumed that the distribution of the new variable $x_{i}$, doesn't correspond with any of the well-known distribution functions. Given a random number $x_{i}^{*}$ from the interval $[0,1]$, the new value of each selected dimension $x_{i}$ is is determined based on the classical mapping function:

$$
x_{i}=h_{x}+\left(1-h_{1}+h_{0}\right) \cdot x_{i}^{*}-h_{0}
$$

where $h_{x}, h_{1}$ and $h_{0}$ are the inputs of the mapping function based on different inputs given by:

$$
\begin{aligned}
& h_{x}=h\left(x=x_{i}^{*}\right) \\
& h_{1}=h(x=1) \\
& h_{0}=h(x=0)
\end{aligned}
$$

Both input and output of the mapping function are always between the range $[0,1]$. The definition of the transformation mapping h-function is the following:

$h\left(\bar{x}_{l}, s_{1}, s_{2}, x\right)=\bar{x}_{l} \cdot\left(1-e^{-x \cdot s_{i 1}}\right)+\left(1-\bar{x}_{l}\right) \cdot e^{-(1-x) \cdot s_{i 2}}$

As illustratively shown in the following figure, the h-function transforms the variable $x_{i}^{*}$ varied randomly with unity distribution to another variable $x_{i}$, which is concentrated around the mean value calculated from the archive. The variation of $\bar{x}_{i}$ implies shifting of the curve between the original lower and upper boundaries of the search range, while the variation of $s_{i, 1}$ and $s_{i, 2}$ affects the bent shape of the curve, i.e. emphasized either exploration or exploitation.

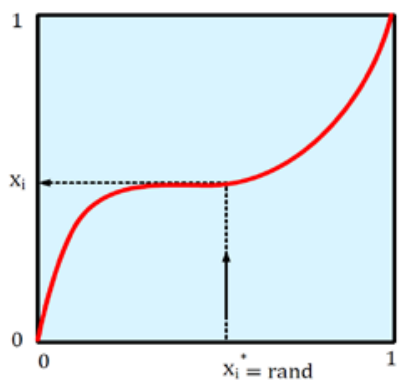

Figure 4. Variable mapping

When the accuracy need to be improved or more global search is required, the factor $f_{s}$ should be increased $\left(f_{s}>1\right)$ and decreased $\left(f_{s}<1\right)$, respectively. Therefore, $f_{s}$ can be used to change the shape of the function.

The above described mapping function does not ensure that the mutation of the optimization variable is performed equitably in both directions (towards min-max bounds). This can adversely affect the global search capability of MVMO (impacting the convergence speed). Thus, a new mapping function is used in this paper:

$$
\begin{array}{cc}
\text { if } x_{i}^{*}<0.5 & \text { if } x_{i}^{*} \geq 0.5 \\
s_{1}^{*}=s_{1} /\left(1-\bar{x}_{i}\right) & s_{2}^{*}=s_{2} / \bar{x}_{i} \\
h_{m 1}=\bar{x}_{i}\left(1-e^{-0.5 \cdot s_{1}^{*}}\right) & h_{m 1}=\left(1-\bar{x}_{i}\right) e^{-0.5 \cdot 5 \cdot s_{2}^{*}} \\
h_{f}=\bar{x}_{i}\left(1-e^{-x_{i}^{*} \cdot s_{1}^{*}}\right) & h_{b}=\left(1-\bar{x}_{i}\right) e^{-\left(1-x_{i}^{*}\right) \cdot s_{2}^{*}}+\bar{x}_{i} \\
h_{c}=2 \cdot x_{i}^{*}\left(\bar{x}_{i}-h_{m 1}\right) & h_{c 1}=2 \cdot h_{m}\left(1-x_{i}^{*}\right) \\
x_{i}=h_{f}+h_{c} & x_{i}=h_{b}-h_{c}
\end{array}
$$

This function ensures that the probability of generating a new value of $x_{i}$ that is greater or smaller than the mean value $\bar{x}_{i}$ is equal. Thus, it allows a higher global search capability, which is extremely important to thoroughly explore the search space and to avoid stagnation in local optima.

\section{RESUlTS}

\section{A. Test System Description}

The far-offshore wind power plant used in this approach is presented in Fig. 6 and corresponds to a real study case. The test system layout is taken from a wind power plant located in Germany, which is connected to the HVDC platform via two AC cables. Detailed information (e.g. parameters) of the wind power plant cannot be provided due to confidentiality reasons. In order to perform the optimization, only the part of the wind power plant up to the PCC is investigated. The nominal total capacity of the connected wind power plant is $288 \mathrm{MW}$ and consists of 48 double-fed induction generator (DFIG), each one with $6 \mathrm{MW}$ rated power. The internal power transmission of the wind power plant is realized by $0.69 / 33 \mathrm{kV}$ transformers, multiple cables with different lengths, and two step up on-load tap-changing transformers of 6.7 MVA and 1555/33/33 kV.

\section{B. Implementation}

In Fig. 5, the general implementation procedure of the optimal reactive power management approach, proposed in this project, is presented. Initially, a multi-layer NN-based wind speed forecasting method is run in MATLAB, from which a 24-hour time series is generated. The reactive power reference of the wind power plant and the tap settings of the transformers are the parameters to be optimized (i.e. optimization variables). Finally, a Python script is used to link the model of the wind power plant built in DigSILENT PowerFactory version 15.2 with MVMO optimization algorithm and obtain the proposed values for the optimization variables throughout MVMO's search.

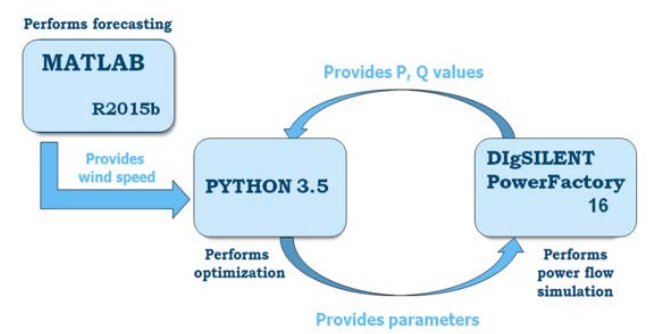

Figure 5. Interaction between MATLAB, Python and DigSILENT PowerFactory 
Considering the output wind speed data of the prediction model, the power produced by each wind turbine is calculated in the Python script by using the following equation:

$$
P_{m}=\frac{1}{2} \cdot\left(\pi \cdot R^{2}\right) \cdot \rho \cdot C_{p} \cdot v_{m}^{3}
$$

Finally, for performing the optimization on 24-hour time horizon, the calculated power is fed into the wind power plant model implemented in DigSILENT PowerFactory.

\section{Optimization results}

The wind scenario for the considered time period of 24-hours is the result of the implemented wind speed forecasting method. The wind profile, shown in the Fig.7, is used for the simulations.

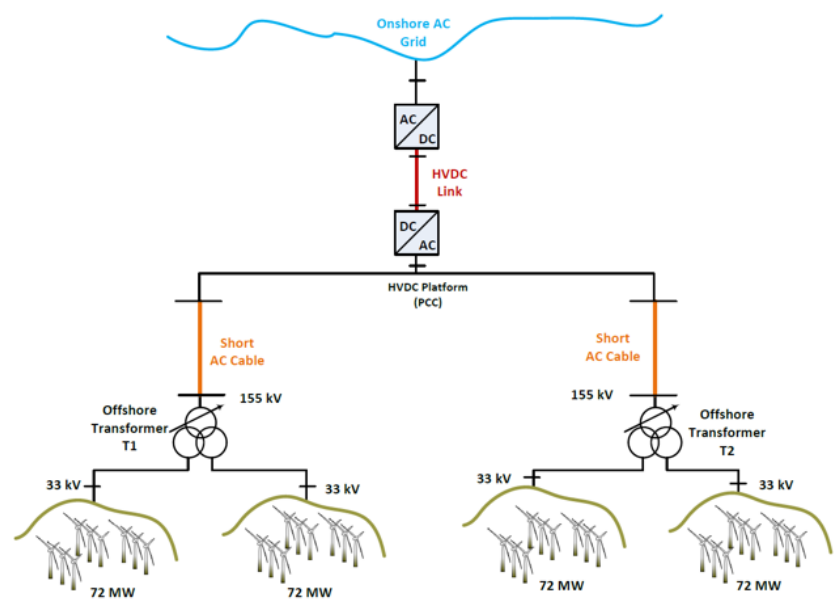

Figure 6. Far-offshore wind power plant layout with HVDC inteconnection link.

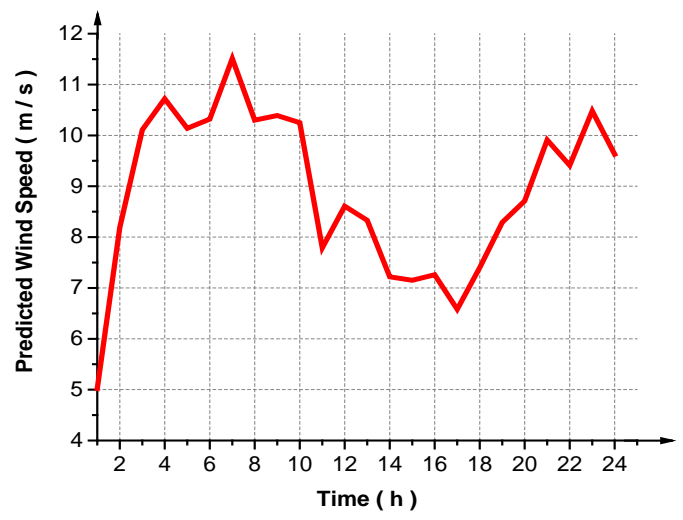

Figure 7. Wind speed variation

In Fig. 8, the difference between the cumulative initial cost, calculated for zero reactive power reference at every wind turbine, and the optimum cost is estimated around $10.97 \%$ for the 24-hour time horizon under investigation. The reactive power set-points for every generating unit derived from the optimization are according to the Grid Code Requirements indicated in [20], since all the values are within the predefined envelope presented in Fig. 9a. Although not shown here due to space constraints, it is worth indicating that the grid code requirement at PCC was also met when the turbines were set to havezero reactive power reference. The figure also evidences that the optimization results in reactive power contributions from each generator according to their individual capabilities and electrical distance. Moreover, it is also confirmed that the normalized search space of MVMO ensures that bound constraints (5) are never violated [4]. This is an advantage with regard to other algorithms, since MVMO does not require extra computing effort to repair solutions to lie within the [min, max] boundaries. Each set of points arranged in the same horizontal line refers to the different value of wind speed. Technical constraints (2)-(4) are handled based on static penalty scheme [21], which proved to be suitable for this problem.

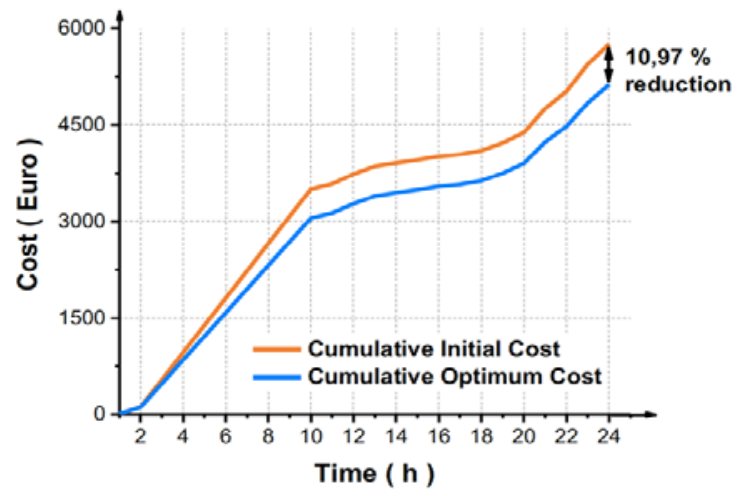

Figure 8. Cumulative cost reduction on 24-hour horizon

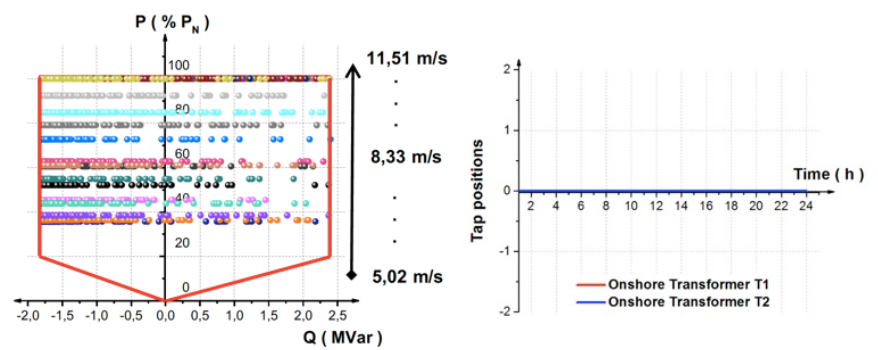

(a)

(b)

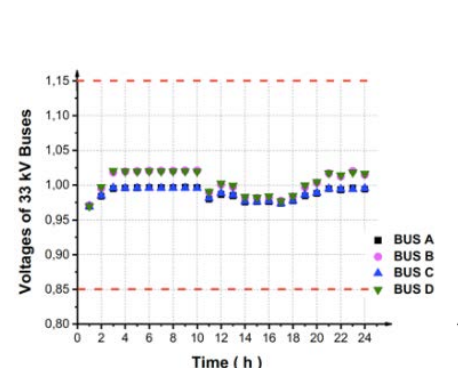

(c)

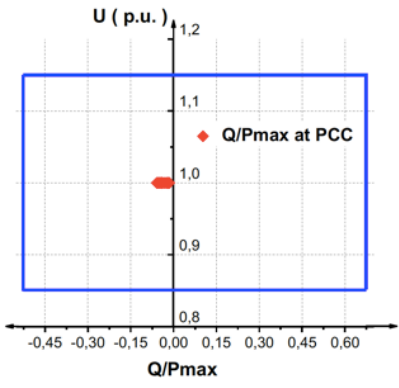

(d)
Figure 9. a) Hourly Q set points of every wind turbine b) On-Load Tap Changer Tap positions - T1 \& T2 c) Voltage levels of 33kV buses d) Reactive power at the PCC

According to Fig. 9d, the reactive power of the HVDC station is maintained within the cabability curve defined for the PCC 
[22]. The reason that all the values are located at the value of 1 p.u. is owed to the consideration of the PCC as a PV bus with constant voltage magnitude. The voltage levels of the $\mathrm{MV}$ - and LV- side of the offshore transformers (33 kV buses) are also within the required range defined by the TSOs [22], as shown in Fig. 9c. Finally, as displayed in Fig.9b, within 24 hours, no tap movements are taking place.

\section{MVMO convergence behavior}

The performance of MVMO for solving the mixed-integer nonlinear complex problem of predictive optimization, within a reduced number of allowed function evaluations, is presented in Fig. 6, where the algorithm converges almost before 300 iterations. The fast convergence behavior and the quick discovery of the optimum solution with minimum risk of premature convergence is revealed thanks to the welldesigned balance between search diversification and intensification of MVMO, which is in agreement with results obtained with the test bed of the 2015 IEEE Competition on Computationally Expensive problems [23].

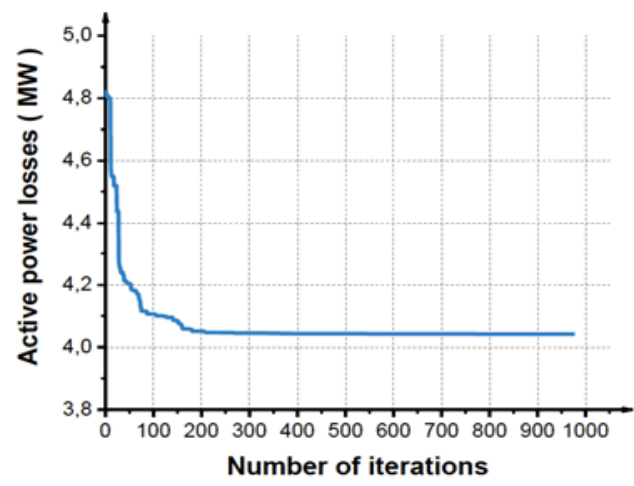

Figure 10. Convergence behavior of MVMO for wind power plant active losses.

\section{CONCLUSIONS}

The main goal of the approach presented in this paper is to minimize the wind power plant power losses, as well as the variations of the transformers tap positions, while the reactive power set-points of individual wind turbines in the power plant are utilizing an optimal reactive power management scheme. The scheme ensures grid code compliance with steady state reactive power requirements, while the operational cost of the wind power plant is also reduced. Finally, it is presented by means of numerical results on a real offshore wind power plant in Germany, that the application of MVMO and it new mapping function in the optimal coordination of reactive power sources in the wind power plant under investigation entails robustness and enhances performance in terms of convergence speed. The application of the presented approach to other wind power plant topologies and Grid Codes is currently under investigation.

\section{REFERENCES}

[1] European Wind Energy Association, "The European offshore wind industry - key trends and statistics 2015,” 2016.

[2] EWEA, "Wind energy scenarios for 2030."

[3] I. Erlich, M. Wilch, and C. Feltes, "Reactive Power Generation by DFIG Based Wind Farms with AC Grid Connection.”
[4] J.L. Rueda, "Metaheuristic Approach for Online Optimal Reactive Power Management in Near-Shore Wind Power Plants," Erasmus Energy Forum, Rotterdam, Netherlands, May 2016..

[5] N. K. Patel and B. N. Suthar, "Optimal reactive power dispatch using particle swarm optimization in deregulated environment," in 2015 International Conference on Electrical, Electronics, Signals, Communication and Optimization (EESCO), 2015, pp. 1-5.

[6] A. R. Bhowmik and A. K. Chakraborty, "Application of Non Dominated Sorting Gravitational Search Algorithm for Solving Optimal Reactive Power Dispatch,” in third IEEE international conference on computation of power, energy, information and communication - ICCPEIC 2014, 2014.

[7] I. Erlich, W. Nakawiro, and M. Martinez, "Optimal dispatch of reactive sources in wind farms," in 2011 IEEE Power and Energy Society General Meeting, 2011, pp. 1-7.

[8] J. A. Momoh, R. Adapa, and M. E. El-Hawary, "A review of selected optimal power flow literature to 1993. I. Nonlinear and quadratic programming approaches," IEEE Trans. Power Syst., vol. 14, no. 1, pp. 96-104, 1999.

[9] J. L. Martinez Ramos, A. Gomez Exposito, and V. H. Quintana, "Transmission power loss reduction by interior-point methods: implementation issues and practical experience," IEE Proc. Gener. Transm. Distrib., vol. 152, no. 1, p. 90, 2005.

[10] V. H. Quintana and M. Santos-Nieto, "Reactive-power dispatch by successive quadratic programming," IEEE Trans. Energy Convers., vol. 4, no. 3, pp. 425-435, 1989.

[11] W. Nakawiro, I. Erlich, and J. L. Rueda, "A Novel Optimization Algorithm for Optimal Reactive Power Dispatch : A Comparative Study."

[12] H. V. Pham, J. L. Rueda, and I. Erlich, "Online Optimal Control of Reactive Sources in Wind Power Plants,” IEEE Trans. Sustain. Energy, vol. 5, no. 2, pp. 608-616, Apr. 2014.

[13] J. L. Rueda and I. Erlich, "Optimal dispatch of reactive power sources by using $\mathrm{MVMO}^{\mathrm{s}}$ optimization," in 2013 IEEE Computational Intelligence Applications in Smart Grid (CIASG), 2013, pp. 29-36.

[14] D. Simon, "Biogeography-Based Optimization," IEEE Trans. Evol. Comput., vol. 12, no. 6, pp. 702-713, Dec. 2008.

[15] S. B. Raha, T. Som, K. K. Mandal, and N. Chakraborty, "Cuckoo search algorithm based optimal reactive power dispatch,” in Proceedings of The 2014 International Conference on Control, Instrumentation, Energy and Communication (CIEC), 2014, pp. 412-416.

[16] S. Biswal, A. K. Barisal, A. Behera, and T. Prakash, "Optimal power dispatch using BAT algorithm,” in 2013 International Conference on Energy Efficient Technologies for Sustainability, 2013, pp. 1018-1023.

[17] R. Rao, "Jaya: A simple and new optimization algorithm for solving constrained and unconstrained optimization problems," Int. J. Ind. Eng. Comput., vol. 7, no. 1, pp. 19-34, 2016.

[18] J. P. S. Catalão, M. I. Pousinho, and V. M. F. Mendes, "An artificial neural network approach for short-term wind power forecasting in Portugal,” Eng Int Syst, vol. 1, pp. 5-11, 2009.

[19] J.L. Rueda, Rueda, and I. Erlich, "Testing MVMO on learningbased real-parameter single objective benchmark optimization problems," in Proc. IEEE Congress on Evolutionary Computation (CEC), pp.1025-1032,Sendai, Japan, May 2015.

[20] Tennet TSO GmbH, "Requirements for Offshore Grid Connections in the Grid of TenneT TSO GmbH,” no. December, p. 14, 2012.

[21] W. Nakawiro, I. Erlich, and J. L. Rueda, "A novel optimization algorithm for optimal reactive power dispatch: A comparative study," in 2011 4th International Conference on Electric Utility Deregulation and Restructuring and Power Technologies (DRPT), 2011, pp. 1555-1561.

[22] Entso-e, "ENTSO-E Draft Network Code on High Voltage Direct Current Connections and DC- connected Power Park Modules," no. April, pp. 1-76, 2014. J. L. Rueda and I. Erlich, "MVMO for Bound Constrained SingleObjective Computationally Expensive Numerical Optimization," in Proc. IEEE Congress on Evolutionary Computation (CEC), pp.1011-1017,Sendai, Japan, May 2015. 